\title{
Characterization of hereditary inclusion body myopathy myoblasts: possible primary impairment of apoptotic events
}

\author{
S Amsili ${ }^{1}$, Z Shlomai ${ }^{2}$, R Levitzki ${ }^{2}$, S Krause ${ }^{3}$, H Lochmuller ${ }^{3}$, H Ben-Bassat ${ }^{2}$ and S Mitrani-Rosenbaum ${ }^{\star, 1}$
}

Hereditary inclusion body myopathy (HIBM) is a unique muscular disorder caused by mutations in the UDP-N-acetylglucosamine 2-epimerase/ $\mathrm{N}$-acetylmannosamine kinase (GNE) gene. GNE encodes a bi-functional enzyme acting in the biosynthetic pathway of sialic acid. Since the underlying myopathological mechanism leading to the disease phenotype is poorly understood, we have established human myoblasts cultures, derived from HIBM satellite cells carrying the homozygous M712T mutation, and identified cellular and molecular characteristics of these cells. HIBM and control myoblasts showed similar heterogeneous patterns of proliferation and differentiation. Upon apoptosis induction, phosphatidylserine externalization was similar in HIBM and controls. In contrast, the active forms of caspase-3 and -9 were strongly enhanced in most HIBM cultures compared to controls, while pAkt, downregulated in controls, remained high in HIBM cells. These results could indicate impaired apoptotic signaling in HIBM cells. Since satellite cells enable partial regeneration of the post-mitotic muscle tissue, these altered processes could contribute to the muscle mass loss seen in patients. The identification of survival defects in HIBM affected muscle cells could disclose new functions for GNE in muscle cells.

Cell Death and Differentiation (2007) 14, 1916-1924; doi:10.1038/sj.cdd.4402208; published online 3 August 2007

Hereditary inclusion body myopathy (HIBM) is a unique neuromuscular disorder characterized by adult-onset, slowly progressive distal and proximal muscle weakness, presenting with an unusual feature, the sparing of the quadriceps. HIBM fibers have typical muscle pathology, including cytoplasmatic rimmed vacuoles and cytoplasmatic or nuclear filamentous inclusions composed of tubular filaments. ${ }^{1}$ The disease is particularly common in the Jewish Persian community (with a prevalence of 1 in 1500), and has been described also worldwide in non-Jewish families, particularly in Japan. ${ }^{2}$ The GNE gene, encoding the bi-functional enzyme UDP-Nacetylglucosamine 2-epimerase/ $\mathrm{N}$-acetylmannosamine kinase (UDP-GIcNAc 2-epimerase/ManNAc kinase), is mutated in all HIBM patients. A single homozygous missense mutation, M712T, located at exon 12 of the gene within its kinase domain, was identified in all Persian and other Middle Eastern Jewish and non-Jewish HIBM patients. ${ }^{3}$ Other missense mutations in GNE have been identified in HIBM patients worldwide. $^{4-7}$ GNE catalyzes two sequential steps in the biosynthetic pathway of sialic acid, ${ }^{8}$ the most abundant terminal monosaccharide on glycoconjugates of eukaryotic cells. ${ }^{9}$ The process by which mutations in this enzyme lead to the disease is not yet understood, and the issue of hyposialylation in HIBM muscles is still controversial. ${ }^{10-12}$ To date, the basic cellular functions of muscles from HIBM patients have not been investigated. Since the outcome in this degenerative disease is loss of muscle mass, analysis of HIBM muscle tissue is necessary to gain more insights in the pathogenic mechanisms underlying HIBM.

In an attempt to evaluate the effects of GNE mutations on HIBM muscle tissue, we have developed primary muscle cultures from HIBM and control muscle biopsies, and used them as an experimental model for the disease. Muscle cultures are established from muscle satellite cells, a population of undifferentiated (UD) mononuclear myogenic cells present in all adult skeletal muscles. Those cells are mitotically quiescent, and start to proliferate in response to various signals in vivo, and when cultured in vitro. ${ }^{13}$ Satellite cells have been used as a model to study myogenesis, cellular development and cellular signaling. ${ }^{14}$ We used those cells to evaluate HIBM muscle cells biology in terms of morphology, proliferation and differentiation abilities, the occurrence of spontaneous and induced apoptotic processes, and the expression of major signaling proteins. Since repair of muscle tissue damage depends on satellite cells activation and functioning, ${ }^{15}$ this cell system might not only reflect the

${ }^{1}$ Goldyne Savad Institute for Gene Therapy, Hadassah Hebrew University Medical Center, Jerusalem, Israel; '2Laboratory of Experimental Surgery, Hadassah Hebrew University Medical Center, Jerusalem, Israel and ${ }^{3}$ Department of Neurology and Gene Center, Friedrich-Baur-Institute, Ludwig-Maximilians-University, Munich, Germany

${ }^{*}$ Corresponding author: S Mitrani-Rosenbaum, Goldyne Savad Institute of Gene Therapy, Hadassah Hebrew University Medical Center, Hadassah Hospital, Mount Scopus, Jerusalem 91240, Israel. Tel: 972-25819134; Fax: 972-25819134; E-mail: stella@cc.huji.ac.il

Keywords: hereditary inclusion body myopathy; HIBM; GNE; muscle satellite cells; apoptosis; Akt

Abbreviations: CMD, congenital myotonic dystrophy; DM, differentiation medium; DMD, Duchenne muscular dystrophy; ERK1/2, extracellularly regulated kinase 1/2; GM, growth medium; GNE, UDP- $N$-acetylglucosamine 2-epimerase/ $N$-acetylmannosamine kinase; GSK-3 $\alpha / \beta$, glycogen synthase kinase-3 $\alpha / \beta$; HD, Huntington disease; HIBM, hereditary inclusion body myopathy; HS, horse serum; IGF, insulin-like growth factor; MyHC, myosin heavy chain; PI, propidium iodide; PS, phosphatidylserine; SMA, spinal muscular atrophy; STS, staurosporine; UD, undifferentiated; UT, untreated

Received 17.1.07; revised 14.6.07; accepted 05.7.07; Edited by G Cossu; published online 03.8.07 
situation in the mature tissue, but also give some clues about the regeneration ability of HIBM muscles, possibly affecting muscle mass loss in patients.

\section{Results}

Establishment of cultures. Cell cultures from muscle biopsies (Table 1) were established, and the myogenic identity of the cultures was assessed at different time points by desmin staining. As illustrated in Figure 1a, more than $90 \%$ cells were positively stained. The cultures were characterized for various biological parameters.

Morphology. Figure $1 \mathrm{~b}$ and $\mathrm{c}$ shows representative phasecontrast microscopy photographs of HIBM and control cultures, before and after differentiation induction, respectively. We could not detect any significant morphologic differences between HIBM and controls. Neither vacuole structures nor inclusion bodies could be detected, with light and electron microscopy (data not shown). Some 'vacuole' structures appeared in the cultures (Figure 1d), but they were rare, were not correlated with the disease status of the donor, and usually disappeared after induction of differentiation.

Proliferation and senescence. The proliferation ability of the cultured myoblasts was analyzed in three matched groups of HIBM and controls (Figure 2a). A total of $5 \times 10^{3}$ cells/well were seeded in microplates, and growth was monitored for 4 weeks by the Automated Microculture Methylene Blue Assay. In the first pair (Figure 2a-1), the growth rate of both HIBM and control cultures was similar. In the second sample (Figure 2a-2), the growth rate of HIBM culture was lower than its two matched controls, and in the third one (Figure 2a-3) it was higher than controls. Since no uniform pattern of HIBM cell proliferation compared to controls was detected, we suggest that there is no correlation between growth rate and disease status. As indicated by Figure 2a-4, both groups are represented in the entire broad spectrum of normal proliferation. Further, to evaluate the renewal potential of the cultured cells, we performed serial

Table 1 Muscle cells characteristics

\begin{tabular}{lccll}
\hline Sample & Gender & Age (years) & Muscle specimen & Status \\
\hline A-1 & F & 30 & Tibialis anterior & HIBM \\
C-1 & F & 28 & Quadriceps & Healthy \\
A-2 & M & 41 & Deltoid & HIBM \\
C-2a & M & 35 & Deltoid & Healthy \\
C-2b & M & 42 & Sartorius & Healthy \\
C-2c & M & 46 & Deltoid & Healthy \\
A-3 & M & 26 & Deltoid & HIBM \\
C-3a & M & 27 & Deltoid & Healthy \\
C-3b & M & 42 & Adductor magnus & Healthy \\
C-3c & M & 20 & Deltoid & Healthy \\
A-4 & F & 47 & Deltoid & HIBM \\
C-4 & F & 42 & Deltoid & Healthy \\
A-5 & M & 29 & Deltoid & HIBM \\
C-5 & M & 33 & Deltoid & Healthy \\
A-6 & F & 35 & Deltoid & HIBM \\
C-6 & F & 43 & Pectoralis & Healthy \\
& & & & \\
\hline
\end{tabular}

passages once a week, until cell divisions stopped and the cultures senesced. Figure $2 b$ shows the maximal number of serial passages the cultures were able to undergo. The present results further illustrate the variability among the cultures.

Differentiation. To evaluate the effect of differentiation induction on the proliferation ability of HIBM cultures and compare it with healthy controls, $5 \times 10^{3}$ cells were seeded in microplates, and grown for 11 days in growth medium (GM). At this time point, considered as 'day 0' in Figure 3a, GM was replaced with differentiation medium (DM), and growth was monitored with the Automated Microculture Methylene Blue Assay for the next 18 days. After 2-4 days cultivation in DM, the mononucleated dividing myoblasts became post-mitotic, and fused to form multinucleated myotubes. As seen in Figure 3a, growth-arrest patterns were similar among the HIBM and control cultures. In all cultures, growth was completely arrested by day 12 in DM. We further analyzed the differentiation capacity of HIBM myoblasts, and determined the expression of myosin heavy chain (MyHC) a

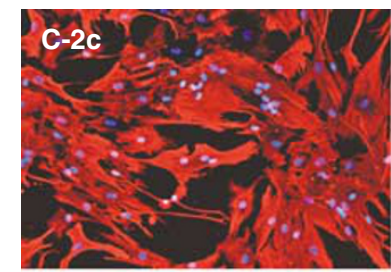

b

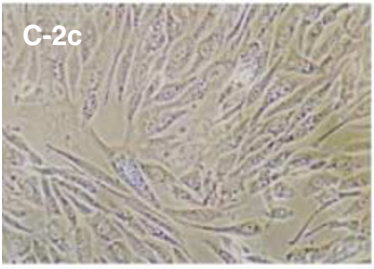

C

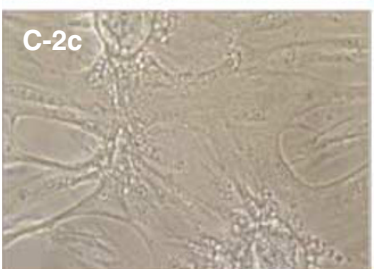

d

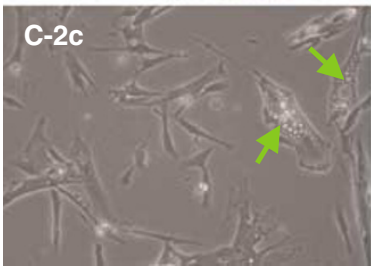

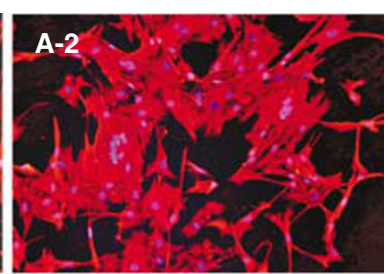
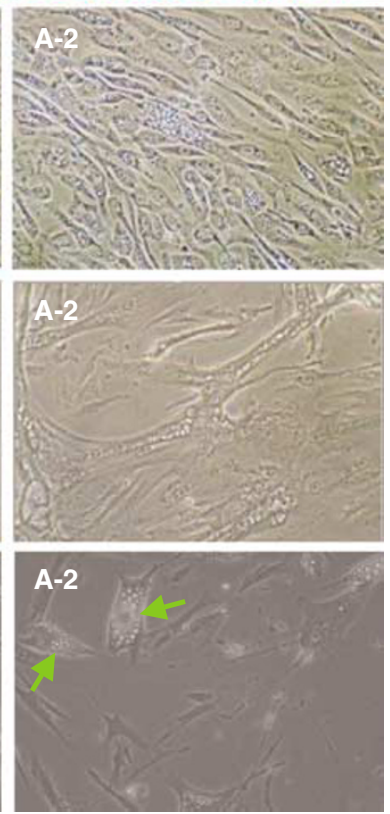

Figure 1 Myogenic identity and morphology of HIBM muscle cultures. (a) Representative desmin staining (Cy3, red) of HIBM and control myoblast cultures. Staining was performed after N-CAM sorting. Nuclei were stained blue by Hoechst dye $(10 \times / 0.3)$. (b and c) Representative phase-contrast microscope photographs of undifferentiated (b) and differentiated (c) HIBM and control cultures $(40 \times / 1.3)$. (d) Representative light microscope photographs of HIBM and control myoblast cultures. Green arrows point at vacuoles structures occasionally observed in some cells $(40 \times 10.75)$ 

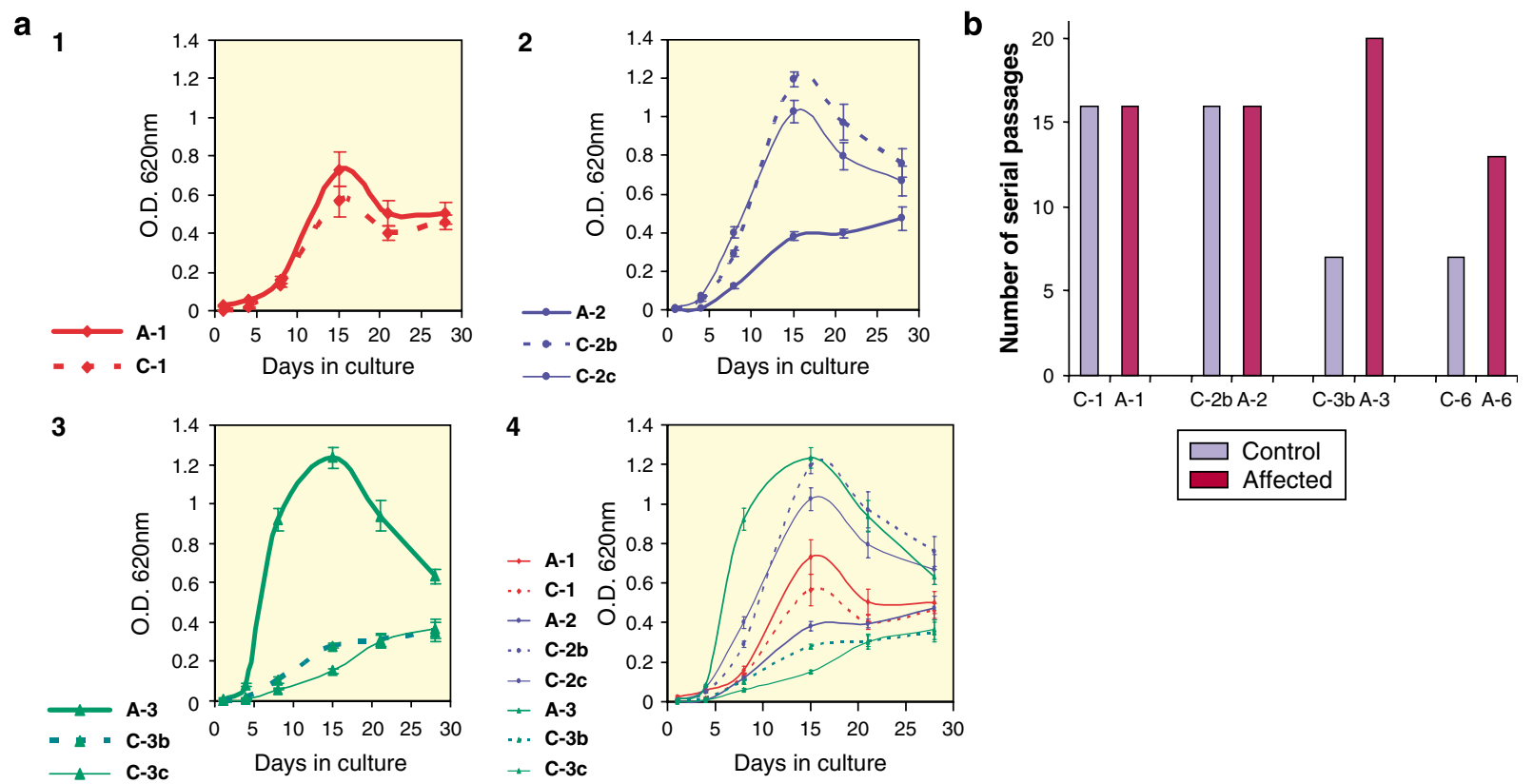

Figure 2 Growth pattern of HIBM muscle cultures. Proliferation (a) and senescence (b) of myoblast cultures. (a) Cells were seeded in GM and growth was monitored for 4 weeks. Each point is the average of eight experiments. Three matched groups of HIBM and control cultures (a1-3) were monitored (HIBM samples in bold line). Figure a4 represents the three groups together. (b) Serial passages of $3 \times 10^{5}$ cells $/ 25 \mathrm{~cm}^{2}$ flasks were performed once a week, till proliferation stopped and the cells degenerated

during differentiation in three pairs of HIBM and matched control cultures, during differentiation (Figure $3 b$ and $c$ ). Cell extracts were prepared before induction of differentiation (Figure 3b-1), and after 1 (Figure 3b-2), 2 (Figure 3b-3) and 5 (Figure $3 b-4$ ) days in DM. Expression of MyHC was analyzed by western blot. As seen in Figure $3 b-1$, some samples differentiated spontaneously, and the expression of MyHC increased between 1 and 2 days in DM (Figure 3b-2 and -3). At this time point, differences were seen among the pairs but with no uniform pattern: in one pair (C-3a, A-3) differentiation started earlier in HIBM than in control, but in the other two pairs it was the opposite. After 5 days in DM (Figure 3b-4), the expression of $\mathrm{MyHC}$ reached a plateau in all six cultures. Therefore, at this time point, cultures were considered as fully differentiated. The fusion index (calculated as the percentage of nuclei within MyHC positively stained cytoplasm) of four matched pairs of cultures, at the same time points during differentiation, gave similar results (data not shown). Figure 3d illustrates the variability in fusion index at the early stages of differentiation. These results indicate that each culture has its own differentiation rate, which is not correlated with the disease status of the donor. It is worth noting that the amount of $\mathrm{MyHC}$ expression in matched fully differentiated cultures is quite concordant within each pair (Figure 3c-4), meaning that the ability of the cells to differentiate is more dependent on muscle type, age and gender of the donors, rather than on their disease status.

Apoptosis. The next experiments were carried out to examine the HIBM cells apoptotic response. The expression level of active caspase- 3 and active caspase- 9 was analyzed by western blot, after treatment of the cells with 0.5 or $2 \mu \mathrm{M}$ staurosporine (STS) for $4 \mathrm{~h}$. This analysis was performed on six pairs of HIBM and control cultures. Figure $4 a$ and $b$ shows the data on four of them. The expression level of the active forms of the two caspases was higher in five out of six HIBM cultures compared to controls. One HIBM sample (A-5) did not show this pattern. This is of interest since the A-5 culture was generated from a muscle biopsy taken at the very early stages of the disease, which did not show pathological changes at that time. Therefore, statistical analysis was performed on five pairs, giving a $P=0.043$. Apoptotic events were further evaluated by FACS analysis of AnnexinV-FITC (apoptotic) and propidium iodide (PI) (dead or necrotic)-stained cells. Since treatment of the cells with 1-10 $\mu \mathrm{M}$ STS for $4 \mathrm{~h}$ did not induce any detectable levels of AnnexinV-FITC staining (data not shown), the cells were treated with $1 \mu \mathrm{M}$ STS for $24 \mathrm{~h}$, as described for a similar analysis of myogenic cells. ${ }^{16}$ As shown in Figure 5a, the four pairs of cultures showed 1-4\% AnnexinV-FITCpositive cells before treatment. All cultures responded to STS, as shown by an increase of up to $10-34 \%$ in positive cells. Variability among the different cultures was not correlated with the disease. However, it is worth noting that there was some similarity within each pair between the HIBM and the control culture (except A-5/C-5), possibly indicating that phosphatidylserine (PS) externalization during apoptosis, assessed by Annexin V-FITC staining, is an intrinsic feature, correlated with age, gender and muscle type. The percentage of PI-stained cells was not significantly altered by STS treatment (Figure $5 b$ and data not shown), but was significantly higher in HIBM cultures compared to matching controls $(P=0.018$ when analyzing six pairs of HIBM and control cultures). We suggest that this difference might represent a lower viability of HIBM cells. The fact that treatment with STS for $24 \mathrm{~h}$ did not increase the number of 


$$
\begin{aligned}
a & \rightarrow A-1 \\
& \rightarrow C-1 \\
& \rightarrow A-2 \\
& \rightarrow C-2 b \\
& \rightarrow C-2 c \\
& \because A-3 \\
& \rightarrow C-3 b
\end{aligned}
$$
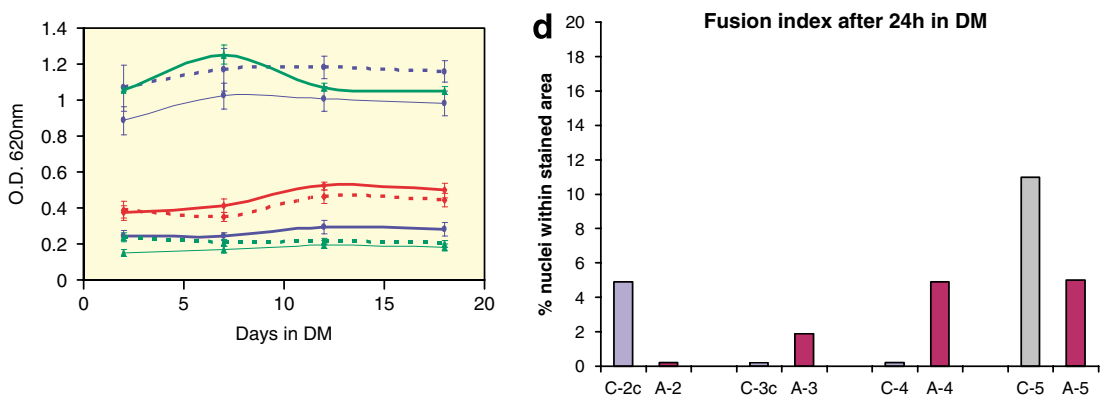

b

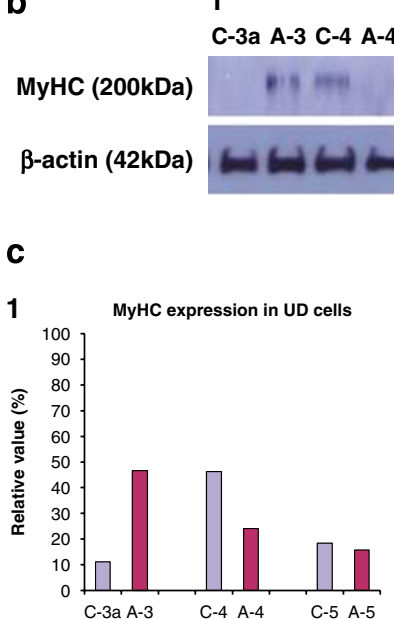

2

C-3a A-3 C-4 A-4 C-5 A-5
3

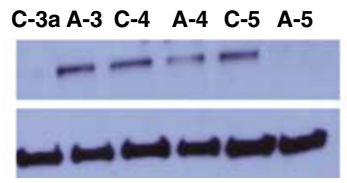

4 C-3a A-3 C-4 A-4 C-5 A-5

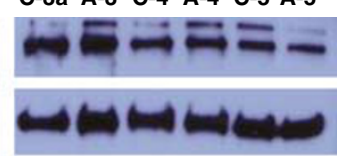

Figure 3 Differentiation profile of HIBM muscle cultures. (a) Growth arrest profile during differentiation. Cells were seeded in GM in microplates. At day 11, considered as 'day 0 ' in the graph, GM was replaced by DM. Each point is the average of eight experiments. The analysis was carried out on three matched groups of HIBM and control cultures, HIBM samples in bold line. (b and $\mathbf{c}$ ) Expression of MyHC during differentiation. (b) Immunoblot analysis was performed on skeletal muscle extracts from HIBM and matched control cultures. Cells were analyzed in GM (UD samples; 1 ), and after 1 (2), 2 (3) and 5 (4) days in DM. Blots were probed with antibodies to MyHC and $\beta$-actin. (c) Each western band was analyzed by densitometry and is represented as the percentage of the corresponding $\beta$-actin value. (d) Fusion index of four matched pairs of HIBM and control cultures after $24 \mathrm{~h}$ in DM. Cells were stained with anti-MyHC antibody (Cy3, red), nuclei were stained blue by Hoechst dye. Fusion index was calculated as the percentage of nuclei within the stained area, out of 600 total nuclei counted

dead cells, suggests that the complete apoptotic cascade of these cells is prolonged.

Signaling pathways. The basal expression level and in vitro kinase activity of Akt was evaluated by Akt kinase assay. Western blot analysis with anti-phospho-glycogen synthase kinase- $3 \alpha / \beta($ GSK $-3 \alpha / \beta)$ revealed similar levels of the phosphorylated substrate in three pairs of HIBM and control cell extracts (Figure 6a), indicating that the expression level of Akt, and its kinase activity when phosphorylated by ATP in vitro, are within the normal range in HIBM cells. To test the Akt phosphorylation level in the cultured cells, we performed western blot analysis on cell extracts (four HIBM and six matched controls), with antiphospho-Akt antibody. In parallel, we analyzed the expression level of pMAPK, compared to the constant level of total MAPK, by the appropriate antibodies. The basal expression levels of the two phosphorylated proteins varied among the samples and from one assay to another, with no correlation to the disease status (data not shown). We further examined the effect of STS treatment on their phosphorylation levels (Figure $6 \mathrm{~b}$ and $\mathrm{c}$ and data not shown). pMAPK expression was highly downregulated after STS treatment in all HIBM and control cells. In contrast, pAkt was highly reduced in control cells, but remained unchanged and high in HIBM cultures $(P=0.01)$.

\section{Discussion}

Mutations in the GNE gene cause HIBM, but the role of the altered GNE protein leading to the muscle-restricted phenotype of the disease is not fully understood. The aim of the present study was to investigate how the most frequent mutation in GNE, M712T, affects the skeletal muscle tissue of patients. The ultimate result of the mutation is loss of muscle mass, but the cellular processes leading to this pathology have not been clarified. To address this issue, we established primary muscle cultures as an experimental tool for HIBM. We examined relevant characteristics of HIBM myoblast cultures, compared with healthy controls, to detect possible changes in HIBM cells biology. Our results indicate that the morphology of HIBM skeletal muscle cultures was similar to controls, both before and after induction of differentiation. No pathological structures were detected within the mononucleated myoblast cells, and not in the multinucleated cells. The 'vacuole' structures occasionally present in some cells did not correlate with the disease status of the donor, and usually disappeared after induction of differentiation. In a recent publication 
Active Caspase3 (17, 20kDa)

$\beta$-actin (42kDa)

2

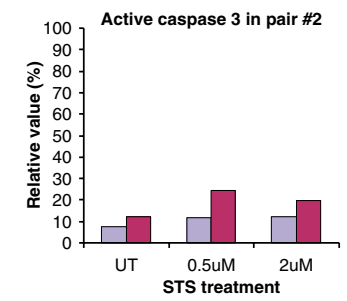

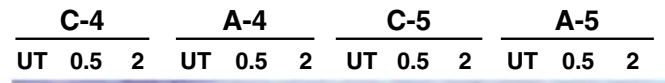
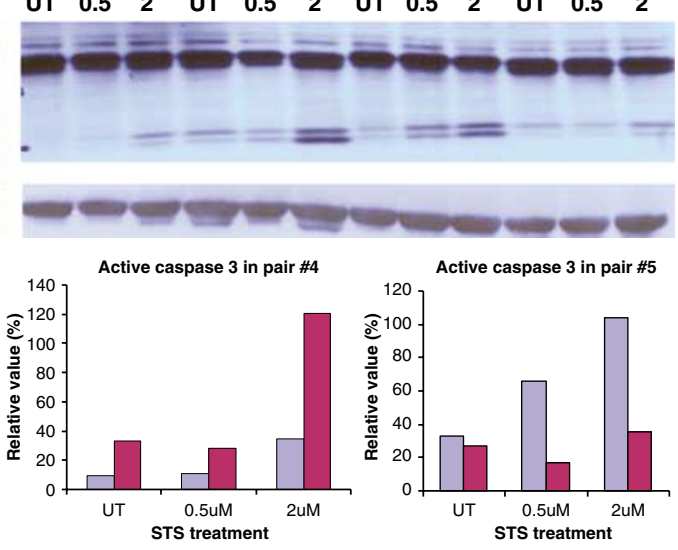

b 1 $\frac{\mathrm{C}-2 \mathrm{a}}{\text { UT } 0.5 \quad 2} \frac{\mathrm{A}-2}{\text { UT } 0.5 \quad 2}$ C-3a A-3

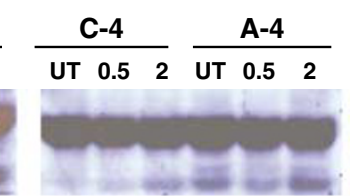

Active Caspase 9 (35kDa)
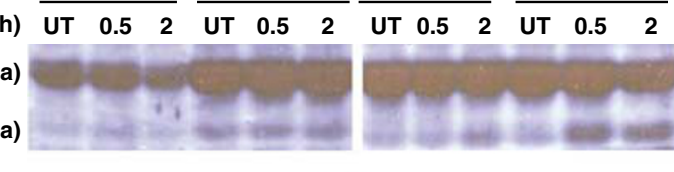

2 $\beta$-actin
$(42 \mathrm{kDa})$
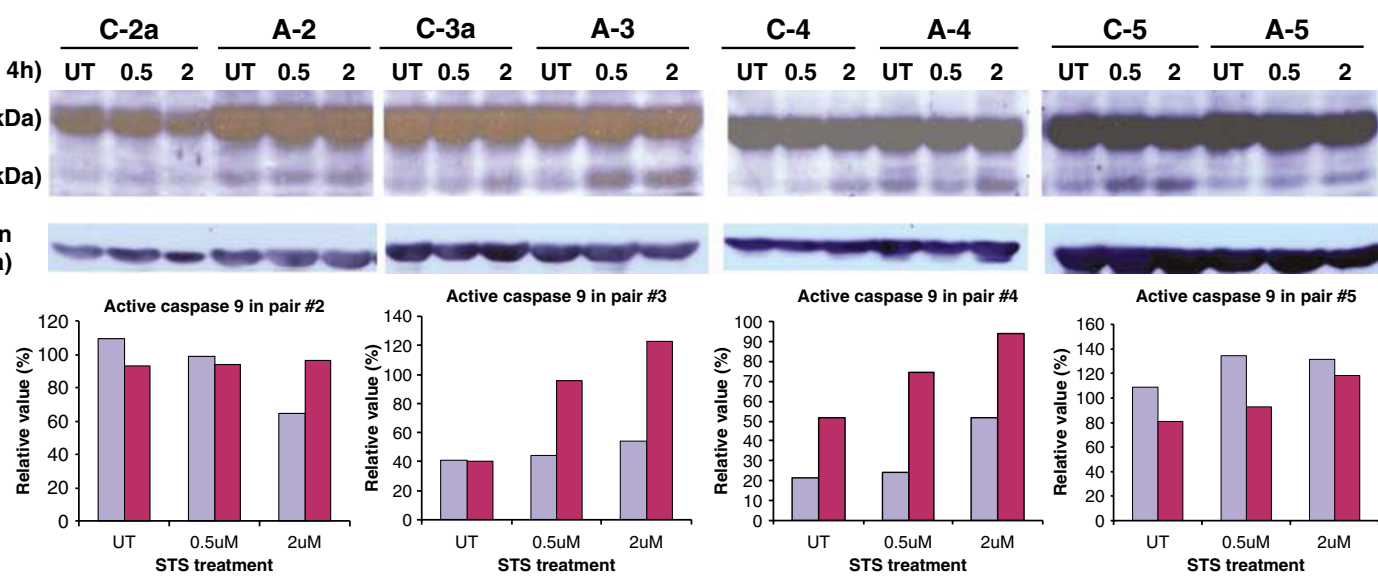

$\square$ Control

Affected

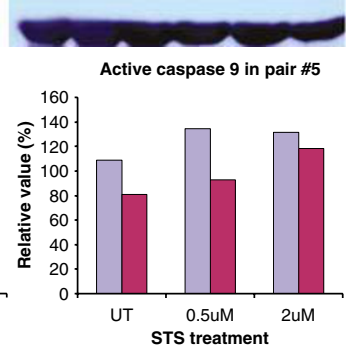

Figure 4 Caspases activation in HIBM muscle cultures. Caspase-3 (a) and caspase-9 (b) expression levels were determined before and after apoptosis induction Apoptosis was induced by treating the cells with 0.5 or $2 \mu \mathrm{M}$ or STS for $4 \mathrm{~h}$. (a1, b1) Immunoblot analysis was performed on HIBM and matched control cultures. Blots were probed with antibodies to caspase-3 or -9 , and with antibody to $\beta$-actin. (a2, b2). Four of the six analyzed pairs are shown. Each band was analyzed by densitometry and is represented as the percentage of the corresponding $\beta$-actin value (UT, untreated, control cultures treated with DMSO only)

referring to human primary muscle cultures from Huntington disease (HD) patients, similar vacuoles were described in HD cultures but not in controls. ${ }^{17}$ As in our cultures, these structures were not visible in myotubes. We suggest that this phenomenon is not an in vitro manifestation of HIBM pathological processes, but a possible characteristic of the normal growth of cultured adult skeletal muscle cells.

The proliferation and differentiation patterns of myoblasts in vitro showed a very broad range, both among controls and HIBM-derived myoblasts. Since impairment of these processes could contribute to the loss of muscle mass observed in HIBM patients, we examined whether HIBM cultures have lower proliferation and differentiation abilities compared to controls. However, the differences in the proliferation capacities of the cultures did not correlate with the disease status: HIBM cultures were all within the growth range of normal controls. The senescence of HIBM cultures, as measured by the number of serial passages the cultures were able to undergo, was also in the range of the normal controls. HIBM myoblasts reacted normally to differentiation induction, in terms of cell division arrest in DM. The differentiation index of HIBM cells, as measured by MyHC expression, both at the cellular level and at the total-protein level was also within the normal range. The variability among the samples at the early stages of differentiation was not correlated with the disease status. Interestingly, in the fully differentiated cultures there was a quite high concordance within each pair of matched samples, possibly indicating some correlation to age, gender and muscle type of the donor. Therefore, it seems that proliferation and differentiation are intrinsic features of the individual samples, and are not correlated with their disease status. In this respect, the HIBM primary myoblast cultures differ from those derived from myotonic dystrophy and HD patients, ${ }^{18}$ that showed lower differentiation index than controls, and from congenital myotonic dystrophy (CMD), ${ }^{19}$ and Duchenne muscular dystrophy (DMD) cells, ${ }^{20,21}$ in which both proliferation and differentiation abilities are damaged. Thus, in these pathologies, basic biological functions of muscle satellite cells in culture are impaired. Interestingly, the fusion index of spinal muscular atrophy I (SMA I) myoblasts is also lower than control cells, but the differentiation abilities of SMA II and SMA III cells were found to be normal. ${ }^{22}$ The differences observed among the three forms of the disease are correlated with its severity, SMA I being the 
a
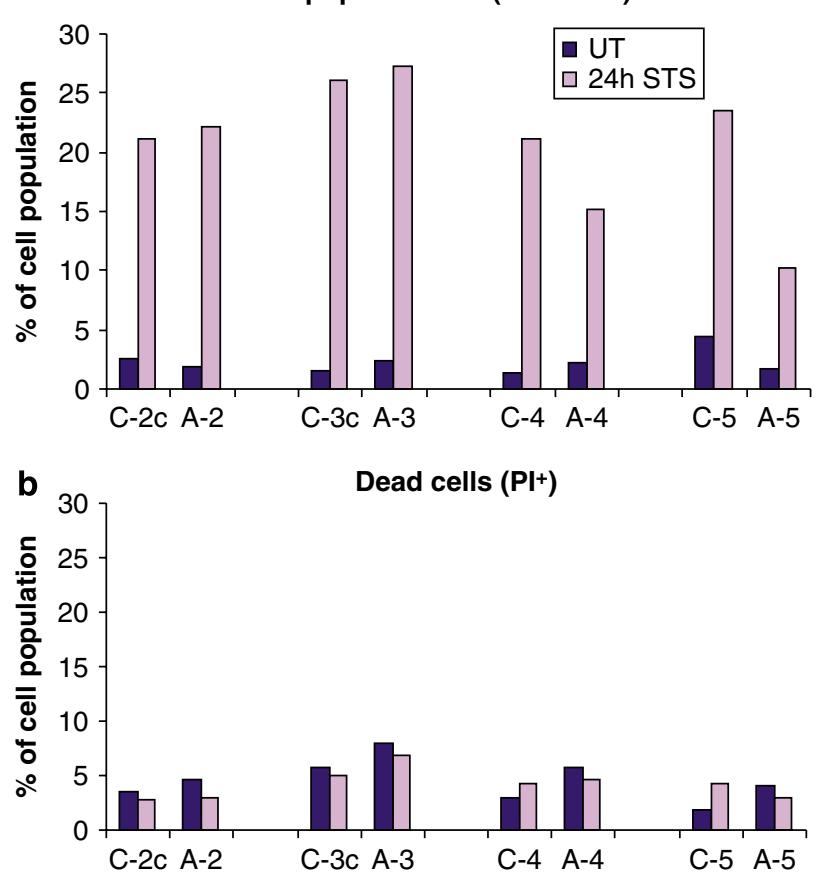

Figure 5 Annexin $\mathrm{V}$ and PI staining of HIBM muscle cultures. Matched pairs of HIBM and control cultures were treated with $1 \mu \mathrm{M}$ STS for $24 \mathrm{~h}$. Cells were collected, stained with the MEBCYTO apoptosis kit, and analyzed by flow cytometry (10000 cells/culture). Annexin V-FITC-positive cells were considered apoptotic, and PI-positive cells as necrotic or dead. Four of the six analyzed pairs are shown

most severe form of the disease, while SMA II and SMA III are milder. This could indicate that in slowly progressive diseases the behavior of satellite cells in culture is close to normal. Our results are consistent with this assumption: indeed, we have found that in HIBM, a slowly progressive disease, proliferation and differentiation of satellite cells are within the normal range, and that impairment of these processes does not contribute directly to the loss of muscle mass observed in HIBM patients. To the best of our knowledge, our research is the first attempt to characterize the basic biological functions of muscle cells in culture from slowly progressive myopathies. The normal rates of proliferation and differentiation preserved in HIBM satellite cells could therefore indicate that those cells retain their regeneration potential. Although very few degenerated/regenerated fibers are observed in HIBM biopsies, ${ }^{23}$ it could be that since muscle loss in HIBM fibers does not occur through necrotic processes, this regenerative potential is not naturally implemented. An alternative explanation for muscle mass loss in patients might be the lower viability of the cells. Cells viability depends on processes leading to cell death, like necrosis and apoptosis. Since necrosis is rarely observed, it is reasonable to assume that the loss of muscle mass in HIBM patients is caused by upregulation of apoptotic processes. In concordance with this hypothesis, apoptotic nuclei and expression of apoptotic proteins were detected in frozen sections of HIBM muscle fibers. ${ }^{24}$ Classical apoptotic characteristics are hardly detected in the multinucleated muscle fiber. It is suggested that during apoptosis only some of the nuclei within each fiber undergo apoptosis at a given time point, the process being very gradual with no drastic effect in the fiber as an entity. ${ }^{25}$ These data raise the possibility that the extent of apoptotic processes in degenerative muscle diseases and particularly in HIBM, is more extensive than previously thought. To investigate this hypothesis, we evaluated the involvement of apoptotic events in HIBM cultures. The expression levels of the active forms of caspase-9 and -3 were found to be higher in three out of four HIBM cultures versus controls. On the contrary, Annexin VFITC staining did not show any consistent difference between four HIBM cultures and their controls. These results could indicate variable sensitivity among the assays and among the culture conditions. Alternatively, it might be that upregulation of caspases activation in HIBM cells does not result in a concordant upregulation of PS externalization, although this apoptotic phenomenon is known to be caspase dependent. ${ }^{26}$ Interestingly, there is accumulating data pointing to caspaseindependent PS externalization occurring during apoptosis, for example in STS-treated primary T lymphocytes, ${ }^{27}$ possibly indicating that in some conditions those two processes may be independently activated. Therefore, it may well be that caspases activation, but not PS externalization, is involved in HIBM pathology. The PI FACS results, showing higher rates of PI-stained HIBM cells, might indicate that caspases upregulation indeed leads to the amplification of death processes in HIBM cells. Since those results suggest that the coordinated control of apoptotic processes might be somewhat impaired, we evaluated the functioning of signaling proteins in HIBM cells. Insulin-like growth factor-I (IGF-I) signaling is involved in muscle atrophy and degeneration, ${ }^{28,29}$ and in the pathogenesis of various neurodegenerative disorders, including S-IBM. ${ }^{30-33}$ Therefore, we evaluated the expression of two major antiapoptotic signaling molecules, activated downstream to IGF receptor: MAPK (extracellularly regulated kinase 1/2 (ERK1/2)), activated via Ras-Raf and Akt/PKB (protein kinase B), activated via phosphatidylinositol 3-kinase (PI3K). ${ }^{34}$ The ERK1/2 cascade promotes cellular proliferation, survival and growth, through processes of DNA synthesis and cell-cycle progression. ${ }^{35}$ Akt is involved in diverse major biological processes, including modulation of cell survival and cell death through apoptosis. ${ }^{36}$ pAkt is known to inhibit caspases activation, ${ }^{37}$ and has been shown to have critical roles in hypertrophy and in the prevention of muscle atrophy in vivo. ${ }^{38}$ The basal expression level of pAkt and pMAPK did not show correlation to the disease status of the donor. As expected, apoptosis induction by STS treatment resulted in downregulation of pMAPK in all cells examined. pAkt was also highly reduced in normal cultures in response to STS; however, it remained high in HIBM cells. These results could indicate that HIBM cells do not respond to STS treatment, but since they showed caspases activation and pMAPK downregulation in response to STS, this possibility is not very likely. Alternatively, it might be that the pathologic enhancement of apoptotic events in HIBM cells (caspases hyperactivation) stimulates antiapoptotic responses in an attempt to overcome death fate, and those preserve the high level of pAkt. Nevertheless, eventually the outcome is increased cell death, as shown by the higher numbers of dead cells in patients' cultures. Indeed, because of pAkt 
pGSK3 $\alpha / \beta(30 \mathrm{kDa})$

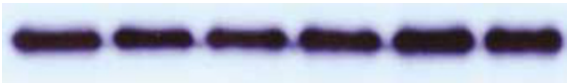

b

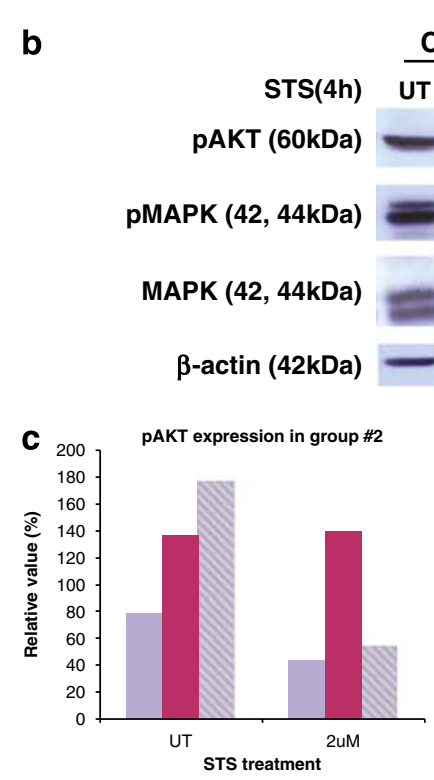

$$
\frac{C-2 b}{\text { UT } 2 \text { UM }} \frac{A-2}{\text { UT } 2 \text { UM }} \frac{\text { C-2c }}{\text { UT } 2 \text { UM }}
$$
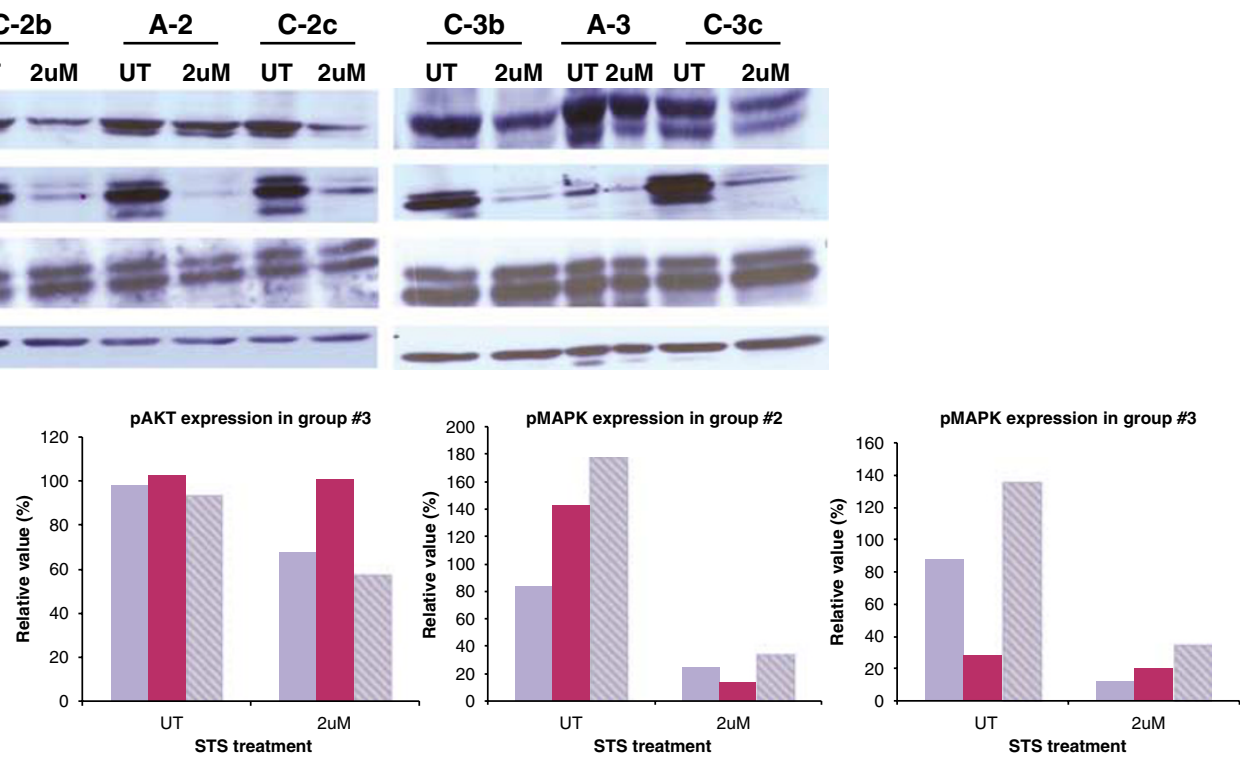

Figure 6 Akt and MAPK activation in HIBM muscle cultures. (a) Akt kinase assay results: pGSK3á/ $\beta$ level after pAkt activation in vitro. Anti-Akt beads were incubated with equal amounts of muscle cell extracts. After binding and washing steps, bound Akt was incubated with ATP and with its unphosphorylated substrate GSK3á/ $\beta$. The proteins were eluted, separated by SDS-PAGE, and analyzed by western blot with anti-phospho-GSK3á/ $\beta$ antibody. (b) Protein levels of pAKT, pMAPK and MAPK were determined before and after apoptosis induction. Apoptosis was induced by treating the cells with $2 \mu \mathrm{M} \mathrm{STS}$ for $4 \mathrm{~h}$. This immunoblot shows the analysis of two HIBM and four matched control cultures (out of the four HIBM and six controls analyzed), with antibodies to pAKT, pMAPK, MAPK and $\beta$-actin. (c) Each band was analyzed by densitometry and is represented as the percentage of the corresponding $\beta$-actin value

multifunctionality, the changes observed in its activation levels could affect not only apoptotic pathways but also other various functions in HIBM muscle cells. However, since these changes were consistent only after apoptosis induction by STS, and other major biological functions remained intact, we assume that the apoptotic sub-pathway of pAKT could be the one involved in the pathophysiology of HIBM. The changes identified in the complex coordinated control of apoptosis in HIBM cells might have only minor impact on the immediate function of affected muscle cells at a given time point, but when persisting for long durations, the accumulated effect, together with some yet unknown changes in signaling pathways, might cause the slow degenerative processes seen in HIBM muscles. The changes in apoptotic processes seen in HIBM satellite cells might be one of the initial pathologic events leading to the degeneration of muscle tissue, since they appear during the mitotic phase, while other biological functions of the cells - morphology, proliferation, senescence and differentiation - seem normal. In contrast, in highly degenerative diseases such as HD, CMD and DMD, most basic biological functions are impaired from the early stages of satellite cells activation, ${ }^{17,19,21}$ and therefore even when apoptotic processes are involved, it is difficult to point to the initial steps of the process. Presently, the upstream events in HIBM pathophysiology, in terms of intracellular and intercellular signaling, have not been elucidated. Further analysis of myogenic signal transduction pathways in HIBM cells is required to better understand the molecular/biochemical mechanisms of the cellular processes leading to HIBM pathology.

In summary, we have established human primary muscle cultures as a cellular tool for characterization of HIBM muscle cells. Our results clearly illustrate the variability among cultures as a problematic feature of the cultured muscle cells, as determined by basic biological criteria of well-matched controls. Nevertheless, we have identified changes related to apoptotic signaling in HIBM skeletal muscle cultures that might be involved in the slow degenerative process and muscle loss of the HIBM adult patients. To better evaluate the involvement of apoptotic pathways in HIBM pathophysiology, these first indications of initial pathological changes in HIBM cultured cells should be further assessed in a larger cohort of patients and eventually in vivo in animal models.

\section{Materials and Methods}

Materials. All chemicals were obtained from Sigma (St. Louis, MO, USA). DME medium, Ham's F-10 medium, FBS, horse serum (HS), PBS, Trypsin, penicillin, streptomycin and glutamine were obtained from Biological Industries (Beit Haemek, |srael).

Subjects and muscle biopsies. Skeletal muscle biopsies were obtained from six clinically and genetically confirmed Jewish Persian HIBM patients, carrying the M712T mutation in GNE, and 10 controls. Table 1 summarizes donor and sample characteristics. Since there is a high variability in many parameters among muscle cultures from different individuals, we matched appropriate controls to each 
HIBM culture, by age, an important factor in muscle cell aging, gender and muscle type. The results of all experiments are displayed individually for each pair or group of matched cultures. These studies were approved by the Institutional Review Board of Wolfson Hospital, Holon, Israel.

Establishment of muscle cell cultures. Some of the cell cultures were obtained from the Muscle Tissue Culture Collection (MD-NET, service structure S1, 01GM0302, BMBF, Eurobiobank) at the Friedrich-Baur Institute. Fresh biopsies were processed, and cell cultures established in rich medium, referred as GM, as described. ${ }^{39}$ To isolate myoblasts from the culture, 2- to 3-week old cultures were sorted by a magnetic cell sorting kit (MACS, Miltenyi Biotech, Auburn, CA, USA) with anti-N-CAM (neural cell adhesion molecule) mAb (clone 5.1H11; LudwigMaximilians University, Munich, Germany). The myogenic identity of the sorted cultures was determined by desmin staining. For this, cells were grown till $70 \%$ confluency, fixed by $3 \%$ paraformaldehyde and permeabilized by $0.5 \%$ Triton-X Incubation with mouse-anti-human desmin antibody (1:150, Dako, Glostrup, Denmark) for $30 \mathrm{~min}$ was followed by incubation with goat-anti-mouse Cy3conjugated secondary antibody $(1: 100$, Jackson ImmunoResearch, West Grove, PA, USA). Nuclei were stained by $15 \mathrm{~min}$ incubation in Hoechst (10 $\mu \mathrm{g} / \mathrm{ml}$; Sigma). All studies were carried out on 4-8 passage cultures.

Differentiation induction. Myoblast cells were grown in GM till $\sim 70 \%$ confluency. To initiate differentiation, GM was replaced by DME medium containing $2 \% \mathrm{HS}$, referred as DM.

Differentiation index. At different time points after differentiation induction (UD, 1, 2, 5 and 7 days), cells were washed with PBS, fixed in methanol for $15 \mathrm{~min}$, and incubated with anti-MyHC antibody (1:5; MF-20 hybridoma, DSHB, lowa city, IA, USA) for $1 \mathrm{~h}$ at room temperature, followed by secondary antibody and Hoechst staining as described above. Fusion index was calculated as the percentage of nuclei within the MyHC-stained area, from a total of 600 nuclei.

Apoptosis induction. Apoptosis was induced in cultures by STS treatment, as described. ${ }^{16,40}$

Flow cytometry. Apoptotic cells were detected by Flow cytometry analysis, using the MEBCYTO Apoptosis Kit (MBL, Nogoya, Japan), according to the manufacturer's instructions. Cell cultures at $\sim 70 \%$ confluence were incubated with $1 \mu \mathrm{M}$ STS for $24 \mathrm{~h}$, collected and stained by Annexin V-FITC and PI. For every sample 10000 cells were counted by FACScalibur (BD, San Jose, CA, USA), and analyzed by the CellQuest Software.

Growth determination. Cell growth was determined by the Automated Microculture Methylene Blue Assay, as described. ${ }^{41}$ To determine the number of passages the cultures are able to undergo, serial passages of $3 \times 10^{5}$ cells in $25 \mathrm{~cm}^{2}$ flasks were performed once a week, till cell divisions stopped and cells degenerated.

Cell lysis. Sub-confluent cell cultures were washed two times with ice-cold PBS and harvested by scraping into ice-cold lysis buffer ( $100 \mathrm{mM}$ Tris- $\mathrm{HCl}$ pH 7.4, $1 \%$ NP-40, $20 \mathrm{mM} \beta$ - mercaptoethanol, $1 \mathrm{mM}$ EDTA, $1 \mathrm{mM}$ PMSF, $17 \mu \mathrm{g} / \mathrm{ml}$ aprotinin, $10 \mu \mathrm{g} / \mathrm{ml}$ leupeptin, $1 \mathrm{mM}$ vanadate, $20 \mathrm{mM} \beta$-glycerophosphate, $20 \mathrm{mM} p$ nitrophenyl phosphate). Lysates were incubated on ice for $30 \mathrm{~min}$, and centrifuged (14000 r.p.m. for $30 \mathrm{~min}$ at $4^{\circ} \mathrm{C}$ ). Supernatants were stored at $-80^{\circ} \mathrm{C}$ until used. Protein concentrations were determined using Bradford Reagent (Sigma).

Western blot analysis. Aliquots of cell lysates $(60 \mu \mathrm{g})$ were separated on $7.5-15 \%$ gradient SDS-PAGE and blotted according to standard procedures. Membranes were incubated with the following primary antibodies: anti-p44/42 MAPK; anti-phospho-p44/42 MAPK, Thr202/Tyr204; anti-phospho-Akt, Thr308 (1:1000; Cell Signaling Technology, Boston, MA, USA); anti-caspase-3 and -9 (1: 100; Santa Cruz Biotechnology, Santa Cruz, CA, USA); anti-MyHC (1:30; MF20 hybridoma supernatant, DSHB) and anti- $\beta$-actin (1:10000; Sigma). Secondary detection was performed with HRP-conjugated antibodies (1:10000; Jackson ImmunoResearch). Protein expression was visualized using the SuperSignal West Pico Chemiluminescent Substrate (Pierce, Rockford, IL, USA). The results were quantified by densitometry using the ImageJ software (NIH, Bethesda, MD, USA). All western analyses were done at least two times.
Akt kinase assay. Akt kinase assay kit (Cell Signaling Technology) was used according to the manufacturer's instructions. Briefly, anti-Akt beads were incubated with equal amounts of muscle cell extracts $(240 \mu \mathrm{g} / 200 \mu \mathrm{l})$. After binding and washing steps, bound Akt was incubated with ATP, for its phosphorylation and activation, and with its un-phosphorylated substrate GSK3 $\alpha / \beta$. Western blot analysis with anti-phospho-GSK-3 $\alpha / \beta$ antibody was performed on eluted proteins.

Microscopic analysis. Light microscope pictures were viewed in BX41 system microscope (Olympus, Tokyo, Japan), objective lenses UPlanFL N $40 \times 1$ 0.75 and $10 \times / 0.3$ (Olympus), photographed by DP70 microscope digital camera and analyzed with the basic software of DP70, DPController (Olympus). Phasecontrast photographs were taken on a LSM410 microscope (Zeiss, Oberkochen, Germany), objective lens Planneofluar $40 \times / 1.3$ (Zeiss). GelMount (Sigma) imaging medium was used. All pictures were taken at room temperature.

Statistical analysis. To compare pairs (HIBMs and controls), the nonparametric Wilcoxon signed-ranks test was applied. This was done for the PI and caspase analyses. The Mann-Whitney non-parametric test was used to compare independent groups of HIBM versus controls. This was carried out for the PAKT analysis, as the numbers of HIBM versus control samples were not the same. A Pvalue of $5 \%$ or less was considered statistically significant.

Acknowledgements. We thank Professor Menachem Sadeh and Dr. Ron Dabby for biopsy material. MF20 antibody, developed by E Butcher, J Lindstrom and DA Fischman, was from the Developmental Studies Hybridoma Bank - DSHB, developed under the auspices of NICHD and maintained by the University of lowa. This work was supported by a grant from the German- Israeli Foundation for Research and Development (GIF), Jerusalem, Israel, and by a grant from the Association Francaise contre les Myopathies (AFM).

1. Argov Z, Yarom R. 'Rimmed vacuole myopathy' sparing the quadriceps. A unique disorder in Iranian Jews. J Neurol Sci 1984; 64: 33-43.

2. Argov Z, Eisenberg I, Mitrani-Rosenbaum S. Genetics of inclusion body myopathies. Curr Opin Rheumatol 1998; 10: 543-547.

3. Eisenberg I, Avidan N, Potikha T, Hochner H, Chen M, Olender T et al. The UDP-Nacetylglucosamine 2-epimerase/ $N$-acetylmannosamine kinase gene is mutated in recessive hereditary inclusion body myopathy. Nat Genet 2001; 29: 83-87.

4. Eisenberg I, Grabov-Nardini G, Hochner H, Korner M, Sadeh M, Bertorini T et al. Mutations spectrum of GNE in hereditary inclusion body myopathy sparing the quadriceps. Hum Mutat 2002; 21: 99-105.

5. Nishino I, Noguchi S, Murayama K, Driss A, Sugie K, Oya Y et al. Distal myopathy with rimmed vacuoles is allelic to hereditary inclusion body myopathy. Neurology 2002; 59: 1689-1693

6. Tomimitsu H, Shimizu J, Ishikawa K, Ohkoshi N, Kanazawa I, Mizusawa H. Distal myopathy with rimmed vacuoles (DMRV): new GNE mutations and splice variant. Neurology 2004; 62: 1607-1610.

7. Broccolini A, Ricci E, Cassandrini D, Gliubizzi C, Bruno C, Tonoli E et al. Novel GNE mutations in Italian families with autosomal recessive hereditary inclusion-body myopathy. Hum Mutat 2004; 23: 632.

8. Hinderlich S, Stäsche R, Zeitler R, Reutter W. A bifunctional enzyme catalyzes the first two steps in $\mathrm{N}$-acetylneuraminic acid biosynthesis of rat liver: purification and characterization of UDP- $\mathrm{N}$-acetylglucosamine 2-epimerase/ $\mathrm{N}$-acetylmannosamine kinase. J Biol Chem 1997; 272: 24313-24318.

9. Schauer R. Sialic acids: fascinating sugars in higher animals and man. Zoology 2004; 107: 49-64.

10. Hinderlich S, Salama I, Eisenberg I, Potikha T, Mantey L, Yarema K et al. The homozygous M712T mutation of UDP- $\mathrm{N}$-acetylglucosamine 2-epimerase/ $\mathrm{N}$-acetylmannosamine kinase results in reduced enzyme activities but not in altered cellular sialylation in hereditary inclusion body myopathy. FEBS Lett 2004; 566: 105-109.

11. Noguchi S, Keira Y, Murayama K, Ogawa M, Fujita M, Kawahara G et al. Reduction of UDP- $N$-acetylglucosamine 2-epimerase/ $N$-acetylmannosamine kinase activity and sialylation in distal myopathy with rimmed vacuoles. J Biol Chem 2004; 279: 11402-11407.

12. Salama I, Hinderlich S, Shlomai Z, Eisenberg I, Krause S, Yarema K et al. No overall hyposialylation in hereditary inclusion body myopathy myoblasts carrying the homozygous M712T GNE mutation. Biochem Biophys Res Commun 2005; 328: 221-226.

13. Charge SB, Rudnicki MA. Cellular and molecular regulation of muscle regeneration. Physiol Rev 2004; 84: 209-238.

14. Al-Khalili L, Kramer D, Wretenberg P, Krook A. Human skeletal muscle cell differentiation is associated with changes in myogenic markers and enhanced insulin-mediated MAPK and PKB phosphorylation. Acta Physiol Scand 2004; 180: 395-403. 
15. Wozniak AC, Kong J, Bock E, Pilipowicz O, Anderson JE. Signaling satellite-cell activation in skeletal muscle: markers, models, stretch, and potential alternate pathways. Muscle Nerve 2005; 31: 283-300.

16. El Fahime E, Bouchentouf M, Benabdallah BF, Skuk D, Lafreniere JF, Chang YT et al. Tubulyzine, a novel tri-substituted triazine, prevents the early cell death of transplanted myogenic cells and improves transplantation success. Biochem Cell Biol 2003; 81: $81-90$.

17. Ciammola A, Sassone J, Alberti L, Meola G, Mancinelli E, Russo MA et al. Increased apoptosis, huntingtin inclusions and altered differentiation in muscle cell cultures from Huntington's disease subjects. Cell Death Differ 2006; 13: 2068-2078.

18. Timchenko NA, lakova $P$, Cai ZJ, Smith JR, Timchenko LT. Molecular basis for impaired muscle differentiation in myotonic dystrophy. Mol Cell Biol 2001; 21: 6927-6938.

19. Furling D, Coiffier L, Mouly V, Barbet JP, St Guily JL, Taneja K et al. Defective satellite cells in congenital myotonic dystrophy. Hum Mol Genet 2001; 10: 2079-2087.

20. Webster C, Blau HM. Accelerated age-related decline in replicative life-span of Duchenne muscular dystrophy myoblasts: implications for cell and gene therapy. Somat $\mathrm{Cell} \mathrm{Mol}$ Genet 1990; 16: 557-565

21. Melone MA, Peluso G, Petillo O, Galderisi U, Cotrufo R. Defective growth in vitro of Duchenne muscular dystrophy myoblasts: the molecular and biochemical basis. J Cell Biochem 1999; 76: 118-132.

22. Arnold AS, Gueye M, Guettier-Sigrist S, Courdier-Fruh I, Coupin G, Poindron P et al. Reduced expression of nicotinic AChRs in myotubes from spinal muscular atrophy patients. Lab Invest 2004; 84: 1271-1278.

23. Argov Z. Hereditary inclusion body myopathy In: Engel AG and Franzini-Armstrong $C$ (eds.). Myology. McGraw-Hill: USA, 2004 pp 1311-1320.

24. Yan $\mathrm{C}$, Ikezoe K, Nonaka I. Apoptotic muscle fiber degeneration in distal myopathy with rimmed vacuoles. Acta Neuropathol 2001; 101: 9-16.

25. Tews DS. Apoptosis and muscle fibre loss in neuromuscular disorders. Neuromuscul Disord 2002; 12: 613-622.

26. Lau A, Arundine M, Sun HS, Jones M, Tymianski M. Inhibition of caspasemediated apoptosis by peroxynitrite in traumatic brain injury. J Neurosci 2006; 26 : 11540-11553.
27. Ferraro-Peyret C, Quemeneur L, Flacher M, Revillard JP, Genestier L. Caspaseindependent phosphatidylserine exposure during apoptosis of primary $\mathrm{T}$ lymphocytes. J Immunol 2002; 169: 4805-4810.

28. Grounds MD. Reasons for the degeneration of ageing skeletal muscle: a central role for IGF-1 signalling. Biogerontology 2002; 3: 19-24.

29. Kandarian SC, Jackman RW. Intracellular signaling during skeletal muscle atrophy. Muscle Nerve 2006; 33: 155-165.

30. Humbert S, Bryson EA, Cordelieres FP, Connors NC, Datta SR, Finkbeiner S et al. The IGF-1/Akt pathway is neuroprotective in Huntington's disease and involves Huntingtin phosphorylation by Akt. Dev Cell 2002; 2: 831-837.

31. Gasparini L, Xu H. Potential role of insulin and IGF-1 in Alzheimer's disease. Trends Neurosci 2003; 26: 404-406.

32. Wilczak N, de Vos RA, De Keyser J. Free insulin-like growth factor (IGF)-I and IGF binding proteins 2, 5 and 6 in spinal motor neurons in amyotrophic lateral sclerosis. Lancet 2003; 361: 1007-1011.

33. Broccolini A, Ricci E, Pescatori M, Papacci M, Gliubizzi C, D'Amico A et al. Insulin-like growth factor I in inclusion-body myositis and human muscle cultures. J Neuropathol Exp Neurol 2004; 63: 650-659.

34. Adams GR. Autocrine and/or paracrine insulin-like growth factor-I activity in skeletal muscle. Clin Orthop 2002; 403S: S188-196.

35. Chang L, Karin M. Mammalian MAP kinase signalling cascades. Nature 2001; 410: 37-40.

36. Abraham E. Akt/protein kinase B. Crit Care Med 2005; 33: S420-422.

37. Kim D, Chung J. Akt: versatile mediator of cell survival and beyond. J Biochem Mol Biol 2002; 35: 106-115.

38. Glass DJ. Skeletal muscle hypertrophy and atrophy signaling pathways. Int J Biochem Cell Biol 2005; 37: 1974-1984.

39. Lochmuller H, Johns T, Shoubridge EA. Expression of the E6 and E7 genes of human papillomavirus (HPV16) extends the life span of human myoblasts. Exp Cell Res 1999; 248: 186-193.

40. McArdle A, Maglara A, Appleton P, Watson AJ, Grierson I, Jackson MJ. Apoptosis in multinucleated skeletal muscle myotubes. Lab Invest 1999; 79: 1069-1076.

41. Oliver MH, Harrison NK, Bishop JE, Cole PJ, Laurent GJ. A rapid and convenient assay for counting cells cultured in microwell plates: application for assessment of growth factors J Cell Sci 1989; 92: 513-518. 